\title{
Treatment of Staphylococcus aureus-induced chronic osteomyelitis with bone-like hydroxyapatite/poly amino acid loaded with rifapentine microspheres
}

\author{
This article was published in the following Dove Press journal: \\ Drug Design, Development and Therapy \\ 20 July 2015 \\ Number of times this article has been viewed
}

\author{
Ling Yan' \\ Dian-Ming Jiang ${ }^{2}$ \\ Zhi-Dong $\mathrm{Cao}^{2}$ \\ Jun $\mathrm{Wu}^{2}$ \\ Xin Wang ${ }^{3}$ \\ Zheng-Long Wang ${ }^{4}$ \\ Ya-Jun $\mathrm{Li}^{5}$ \\ Yong-Fen $\mathrm{Yi}^{\prime}$ \\ 'Department of Pathology, College \\ of Basic Medicine, ${ }^{2}$ Department \\ of Orthopaedic Surgery, The First \\ Affiliated Hospital, ${ }^{3}$ Pediatric Hospital, \\ ${ }^{4}$ Department of Cardiology, The First \\ Affiliated Hospital, ${ }^{5}$ Department of \\ Radiology, College of Basic Medicine, \\ Chongqing Medical University, \\ Chongqing, People's Republic of China
}

Correspondence: Yong-Fen Yi Department of Pathology, College of Basic Medicine, Chongqing Medical University, No. I Xueyuan Road, Yuzhong District, Chongqing 4000I6, People's Republic of China

Tel +86 I3 $7083 \quad 10802$

Fax +862368485540

Email yiyongfen1953@aliyun.com
Purpose: The purpose of this study was to investigate the curative effect of bone-like hydroxyapatite/poly amino acid (BHA/PAA) as a carrier for poly(lactic-co-glycolic acid)-coated rifapentine microsphere (RPM) in the treatment of rabbit chronic osteomyelitis induced by Staphylococcus aureus.

Methods: RPM was prepared through an oil-in-water emulsion solvent evaporation method, and RPM was combined with BHA/PAA to obtain drug-loaded, slow-releasing materials. Twenty-six New Zealand white rabbits were induced to establish the animal model of chronic osteomyelitis. After debridement, the animals were randomly divided into three groups $(n=8)$ : the experimental group (with RPM-loaded BHA/PAA), the control group (with BHA/PAA), and the blank group. The RPM-loaded BHA/PAA was evaluated for antibacterial activity, dynamics of drug release, and osteogenic ability through in vitro and in vivo experiments.

Results: In vitro, RPM-loaded BHA/PAA released the antibiotics slowly, inhibiting the bacterial growth of $S$. aureus for up to 5 weeks. In vivo, at week 4, the bacterial colony count was significantly lower in the experimental group than in the control and blank groups $(P<0.01)$. At week 12, the chronic osteomyelitis was cured and the bone defect was repaired in the experimental group, whereas the infection and bone defect persisted in the control and blank groups.

Conclusion: In vitro and in vivo experiments demonstrated that RPM-loaded BHA/PAA effectively cured $S$. aureus-induced chronic osteomyelitis. Therefore, BHA/PAA has potential value as a slow-releasing material in clinical setting. Further investigation is needed to determine the optimal dosage for loading rifapentine.

Keywords: osteomyelitis, antibiotics-loaded bone materials, bone defect, antimicrobial, osteoconduction

\section{Introduction}

Chronic osteomyelitis is mainly caused by Staphylococcus aureus, ${ }^{1}$ in particular, methicillin-resistant S. aureus (MRSA). Despite the progress and development of surgical techniques and antibiotic drugs, ${ }^{2}$ it is difficult to treat chronic osteomyelitis in clinical setting. With traditional treatment methods, it is difficult to achieve effective bactericidal drug concentrations at the infection site through intravenous or oral antibiotics. In addition, it is difficult for the patients to accept the treatment because it is prolonged and accompanied by high toxicity and adverse side effects.

In the $1970 \mathrm{~s}$, Buchholz and Engelbrecht ${ }^{3}$ and Wahlig et $\mathrm{al}^{4}$ reported that treatment of osteomyelitis through the application of gentamicin combined with poly-methylmethacrylate (PMMA) bone cement at the bone infection site resulted in 
significant improvements. However, PMMA bone cement does not degrade and thus has many disadvantages: 1) it releases a large amount of heat in the polymerization process, which leads to some antibiotic failures; ${ }^{5}$ 2) it lacks the nano-microporous structure of the natural bone, so antibiotics are not completely released, which partially neutralizes the antibiotic effect; ${ }^{6}$ ) it cannot be degraded after implantation in the body, which requires a secondary operation to take out PMMA and reconstruct the bone defects; and 4) it can induce secondary infection. ${ }^{7}$

The development of tissue bioengineering and forging technologies has resulted in many new releasing materials that have good biocompatibility, are biodegradable, and are capable of osteogenesis, which can be used to replace the PMMA bone cement, such as hydroxyapatite (HA), chitosan, and synthetic polymers. One of the most studied materials is HA. Because HA constitutes the main component of bone, it has good biocompatibility and biological activity and is widely used for bone tissue repairing and as a replacement material. ${ }^{8,9}$

The local placement of biodegradable, slow-releasing antibiotic materials is considered the most effective treatment method for osteomyelitis. ${ }^{10,11}$ The carrier provides a sustained release of antibiotics at the infection site to eradicate the bacterial infection and avoid the side effects of systemic drugs. Simultaneously, as the drug is released and the material is gradually degraded and absorbed, the material induces and promotes new bone formation to reconstruct the bone defect, which avoids the second reconstruction of bone defect. ${ }^{12}$

Bone-like hydroxyapatite (BHA) is carbonated nanohydroxyapatite (n-HA) and is similar to a normal bone structure. It exhibits good biodegradation performance, biocompatibility, and osteogenic activity, and its biological activity is stronger than that of $n-H A .{ }^{13}$ Good biocompatibility and bone conduction of BHA combines with good mechanical performance and processability of poly amino acid (PAA) to create a potential BHA/PAA bone repair material.

Poly(lactic-co-glycolic acid) (PLGA) has good biocompatibility, safety, and biodegradability, and can be easily made into clusters or membranes. In many studies, PLGA was used as a carrier material for the controlled drug release. ${ }^{14}$

Rifapentine (RPT) is a derivative of rifampicin that has an antibacterial spectrum similar to that of rifampin, a stronger antibacterial activity, and a drug elimination half-life that is longer than that of rifampin. Its ability to penetrate is stronger than that of vancomycin, ${ }^{15}$ and it can penetrate into infected bone, dead bone, biofilm, and inflammatory cells to effectively eradicate bacterial infections.
This study used BHA/PAA as the carrier of rifapentine microsphere (RPM). In vitro and in vivo, various assays to determine the drug releasing ability, antibacterial activity, and osteogenesis induction ability of RPT-loaded BHA/PAA were conducted, which provide a new approach for the clinical treatment of chronic osteomyelitis.

\section{Materials and methods Preparation of materials}

RPM was prepared through an oil-in-water emulsion solvent evaporation method. Briefly, $200 \mathrm{mg}$ of PLGA was dissolved in $10 \mathrm{~mL}$ of methylene chloride, and $50 \mathrm{mg}$ of RPT was dissolved in the polymer solution. RPMs were prepared through an oil-in-water emulsion solvent evaporation method, with a coating rate of $85.78 \% \pm 2.00 \%$, a loading drug dose of $17.16 \% \pm 0.40 \%$, and a mean diameter of $25.26 \pm 5.45 \mu \mathrm{m}$.

The BHA/PAA materials, A0 (no drug), were prepared using the standard atmospheric pressure solution method. ${ }^{16}$ RPMs of different RPT doses were composited with BHA/ PAA to make RPM-loaded materials A1 and A2. The RPT doses of materials $\mathrm{A} 1$ and $\mathrm{A} 2$ were $4 \mathrm{wt} \%$ and $2 \mathrm{wt} \%$, respectively. The three wafer materials were $8 \mathrm{~mm}$ diameter, $1 \mathrm{~mm}$ thick, and weighed $100.48 \pm 1.45 \mathrm{mg}$.

Using the same method, we prepared cylindrical BHA/ PAA and RPM-loaded BHA/PAA, with size $15 \times 5 \times 5 \mathrm{~mm}^{3}$, weight $750.50 \pm 8.54 \mathrm{mg}$, and aperture size $100-500 \mu \mathrm{m}$. The RPT dose of RPM-loaded BHA/PAA was $30 \mathrm{mg}$ (4 wt\%). The materials and testing data were from the Sichuan University Material Analysis Inspection Center and the Sichuan University National Nano-Materials Incubation Base. The materials mentioned earlier were disinfected by low-temperature plasma.

\section{In vitro experiment}

\section{Determination of releasing profile and antibacterial} activity

The releasing profile and antibacterial activity of RPM-loaded BHA/PAA was determined in vitro. We used the three kinds of wafer materials, ie, A0, A1, and A2 for the bacterial inhibition assay. Each of the three kinds of wafer materials and RPT drug-sensitive piece was inoculated onto $90 \mathrm{~mm}$ agar medium coated with $S$. aureus and Escherichia coli (concentration of approximately $3 \times 10^{8} \mathrm{CFU}$ (colony forming units)/ $\mathrm{mL}$ ) and incubated at $37^{\circ} \mathrm{C}$ in a constant-temperature incubator for 1 day. Then, the bacterial inhibition zones were measured, and the materials were taken out 
and replaced on the new medium with $S$. aureus and E. coli being continuously cultivated (the RPT drug-sensitive piece was used on the first day as a positive reference only). Every 3 days, the diameter of the inhibition zone was measured and recorded, and the culture media were replaced. Finally, the change in the antibacterial activity over time was determined through the disappearance of the bacterial inhibition zone. This experiment was repeated five times for $S$. aureus and E. coli.

For the antibacterial experiment, each of the three kinds of wafer materials, ie, A0, A1, and A2 was placed on 24-hole culture plates containing $S$. aureus and E. coli (concentration of approximately $3 \times 10^{8} \mathrm{CFU} / \mathrm{mL}$ ) in under the standard incubation conditions $\left(37^{\circ} \mathrm{C}, 5 \% \mathrm{CO}_{2}, 5 \%\right.$ air). After 5 hours, the wafer materials were taken out and processed as follows: fixation, dehydration, substitution, critical point drying, and gold spraying. Finally, under the conditions of high vacuum and $20 \mathrm{kV}$, the anti bacterial effects were observed through scanning electron microscopy (SEM) by examining the adhesion and surface growth of the bacteria on the material. This experiment was repeated three times.

\section{Determination of osteogenic ability in vitro}

The BHA/PAA wafer materials were cleaned three times with PBS and then placed in the 24-hole culture plate. MG63 cells $\left(3 \times 10^{4}\right.$ cells/hole $)$ were incubated under the standard environmental conditions $\left(37^{\circ} \mathrm{C}, 5 \% \mathrm{CO}_{2}, 5 \%\right.$ air). Cell adhesion and growth was observed under inverted microscope. After inoculation for 7 days, one wafer was taken out and processed as described earlier for SEM analysis. MG63 cells on the material surface were observed through SEM for adhesion, growth, and calcium nodule formation.

\section{In vivo experiment}

Animal models with chronic osteomyelitis were established and treated. All the surgical procedures were approved and performed by the Animal Care Committee of Chongqing Medical University in the People's Republic of China. We used 26 New Zealand white rabbits that were 4 months old and weighed $2.5-3.0 \mathrm{~kg}$ (an average of $2.73 \mathrm{~kg}$ ). After breeding and observation for 5 days, the New Zealand rabbits were given pentobarbital $(30 \mathrm{mg} / \mathrm{kg})$ anesthesia intravenously in the ear margin and then were placed on the operating table. The right side of the medial upper tibia was shaved and disinfected with povidone-iodine solution. An approximately $2 \mathrm{~cm}$ long, proximal tibial medial, longitudinal incision was made to expose the tibial metaphysis. A $4.5 \mathrm{~mm}$ Kirschner wire was inserted into the medullary cavity, letting out a small amount of bone marrow. Approximately, $0.2 \mathrm{~mL}$ of $5 \%$ sodium morrhuate (as scleromate; ${ }^{17}$ Shanghai Donghai Pharmaceutical Factory, Shanghai, People's Republic of China) was injected through this hole. After 5 minutes, $0.2 \mathrm{~mL}$ of $3 \times 10^{8} \mathrm{CFU} / \mathrm{mL}$ ATCC25923 S. aureus suspension was injected into this aperture. Then, a syringe with a small amount of saline was used to flush the residual bacteria into the injection hole, to ensure that all bacteria entered into the medullar cavity. The hole was filled with an approximately $4.5 \mathrm{~mm}$ diameter foreign cotton ball. Finally, the hole was immediately closed with bone wax to prevent bacterial leakage into the surrounding soft tissues. The wound was washed and then closed with layered sutures.

Four weeks after the induction of osteomyelitis, in the rabbit tibia, osteomyelitis was confirmed by X-ray according to the descriptive criteria of Norden et $\mathrm{al}^{18}$ and Pathological HE staining to use the descriptive criteria of Smeltzer et $\mathrm{al}^{19}$ and the G-staining and coagulase tube test of culture bacteria of lesion bone tissue. Two rabbits were conducted for pathological hematoxylin and eosin (HE) staining to evaluate the presence of osteomyelitis inflammation. The remaining 24 rabbits were treated according to the previously stated surgical operations for the removal of necrotic, hardening, and infected soft and bone tissue. After the infected bone and soft tissue were cleaned, the wounds were washed with $2 \%$ hydrogen peroxide solution and saline. The 24 animals were then randomly divided into three groups $(\mathrm{n}=8)$ : the experimental group received $15 \times 5 \times 5 \mathrm{~mm}^{3}$ RPM-loaded BHA/PAA material, the control group received $15 \times 5 \times 5 \mathrm{~mm}^{3}$ BHA/PAA (unloaded) material, and the blank group received only debridement without filling with any material. The wounds were washed with saline and closed with layered sutures. The animals were kept postoperatively in individual cages, fed a routine diet, and allowed unrestricted activity.

Postoperatively, the animals were observed for the following conditions: diet, hair, activities, gait, and wound. The relevant indicators that were detected pre- and postoperatively were weight, white blood cell (WBC) count, and C-reactive protein changes.

The radiological evaluation of chronic osteomyelitis was performed 4 weeks after induction, and the chronic osteomyelitis established in the rabbit tibia was determined using radiographs. To verify the presence of osteomyelitis, two radiologists independently evaluated the radiographs in a double-blind manner using the criteria described by Norden et al. ${ }^{18}$ At a different postoperative time point after the debridement and treatment of chronic osteomyelitis, X-ray 
was used to evaluate the presence of bone lesion changes, degradation and absorption of the implant material, and new bone formation.

Pathological and immunohistochemical examination was performed 4 weeks after the induction of osteomyelitis. The bone tissue lesions of two sacrificed animals and from all animals after debridement surgery were collected and processed as follows: fixation with 10\% formaldehyde solution, decalcification, paraffin embedding, sectioning into 5- $\mu \mathrm{m}$-thick slices, and staining with HE. To verify the histologic diagnosis of chronic osteomyelitis, two pathologists independently evaluated the samples in a double-blind manner. Twelve weeks after the debridement and treatment, the animals were sacrificed, and bone tissue specimens from each group were collected for pathological HE staining and immunohistochemical staining of collagen fibers in hard tissue slices to evaluate the inflammation and osteogenesis changes of chronic osteomyelitis.

For micriobial examination, samples were collected from the bone tissue lesion, immediately stored in sterile tubes, and sent to the laboratory. Then samples were inoculated on blood agar plates for bacterial culture. The cultured bacteria were subjected to G-staining and coagulase tube test to detect the presence of $S$. aureus. In addition, 4 weeks after the debridement and treatment, four animals were sacrificed in each group by giving an overdose of sodium pentobarbital. Bone tissue lesions were collected and pulverized, and then, $1 \mathrm{~g}$ of bone sample was precisely weighed and mixed with $1 \mathrm{~mL}$ of saline to prepare a suspension. After tenfold serial dilution with saline, $0.1 \mathrm{~mL}$ of the diluted sample was inoculated onto blood agar plates and incubated at $37^{\circ} \mathrm{C}$ for 48 hours. Finally, colony counts per gram of bone tissue were calculated. All tests were carried out in triplicate and under aseptic conditions.

\section{Statistical analysis}

The experimental data were analyzed using the SPSS18.0 statistical software package, and the data were expressed as the mean \pm standard deviation $(\overline{\mathrm{x}} \pm \mathrm{s})$. The differences between groups were analyzed using Student's $t$-test. $P$-values $<0.05$ were considered to be statistically significant.

\section{Results}

\section{In vitro experiment}

The bacterial inhibition assays showed that the maximum inhibition zones in the medium containing $S$. aureus and E. coli were $23.25 \pm 0.24$ and $18.42 \pm 0.38 \mathrm{~mm}$, respectively. The inhibition effect lasted for 5 weeks and 4 weeks in the medium containing $S$. aureus and E. coli, respectively (Figure 1).
For antibacterial experiment assay, materials A0, A1, and A2 were cultivated with $S$. aureus and $E$. coli for 5 hours. Via SEM observation, there were a large number of bacteria adhesion and growth adhered on the materials with no drugloaded. The bacterial adhesion and growth clearly decreased with the drugs-loaded materials. The decrease was dosedependent and depended on the doses of the loaded drug (Figures 2 and 3).

MG63 cells that were cultivated together with BHA/ PAA for 1 week were observed under inverted microscope and SEM. Cell adhesion on the material was good, and there was extensive growth with the formation of many calcium nodules (Figure 4).

\section{General observations after osteomyelitis induction, debridement, and treatment}

Within 1 week of osteomyelitis induction and the debridement surgery, it was observed that the hair of all animals had become coarse and the limbs on the side of the surgery dangled without touching the ground. Within 3 days after the surgery, the animals became lethargic, and all animals consumed less than normal. However, this change in behavior gradually recovered after 1 week. Three days after osteomyelitis induction, the wound was red and had swelling. After 10-14 days, the wound formed fistulas and had discharge, which leaked until the debridement surgery was performed. One week after the debridement surgery, the early redness and swelling of the lesions in the experimental group showed improvement. After 2 weeks, the normal gait was restored and the hair of the animals was smooth and comparable to the preoperative condition. In the control and blank groups, the redness and swelling continued for 3 weeks and then gradually improved with persistent mild swelling at the local site.

\section{Evaluation of weight changes and relevant laboratory indicators}

After osteomyelitis induction, the weights of the animals initially decreased and then later increased, but were still lower than normal values. 1 week after the debridement surgery, the weights of the animals gradually increased in the drugloaded group, and after 2 weeks, the weights had largely returned to normal. In the control and blank groups, the weights slowly increased, but were still lower than normal at week 4 . WBC count and C-reactive protein analysis were performed. The WBC count of normal rabbits was approximately $8.0 \times 10^{9}$ cells $/ \mathrm{L}$. The WBC count was notably high $\left(14.25 \pm 1.32 \times 10^{9}\right.$ cells/L) on the day before the debridement surgery because of the presence of osteomyelitis. Within the 

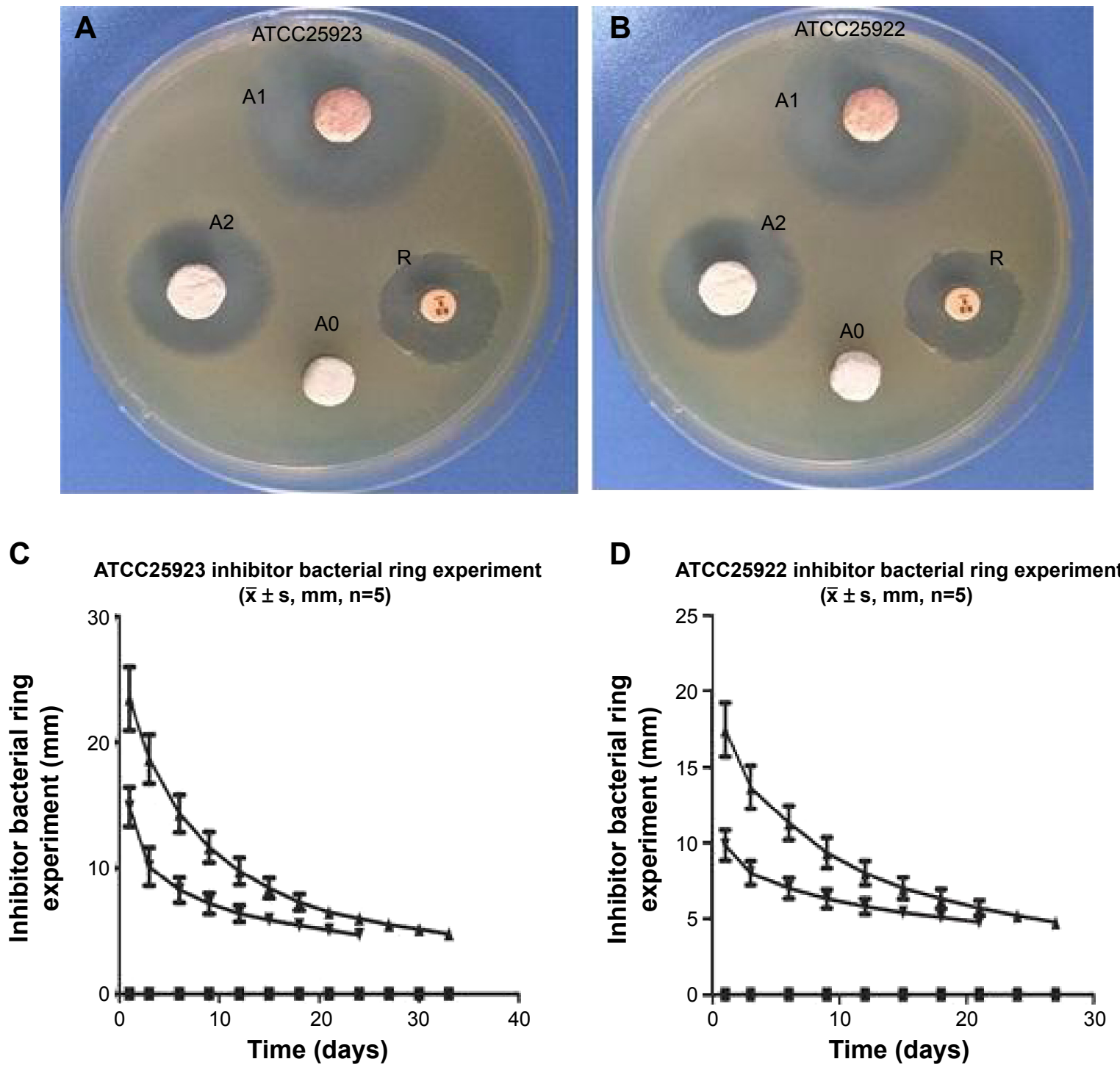

Material A0 $\neq$ Material A1 + Material A2

Figure I The bacterial inhibition experiments.

Notes: (A) and (B) are the maximal bacterial inhibition zones (diameter) on day I with strains ATCC25923 and ATCC25922, respectively. A0, no loading drug; AI, 4\% RPT, A2, 2\% RPT, R, the sensitive drug piece of RPT. (C) and (D) are mean diameters of bacterial inhibition changes of ATCC25923 and ATCC25922 strains, respectively, with time.

Abbreviation: RPT, rifapentine.

first 3 days after the debridement surgery, the WBC counts of the three groups were elevated above the preoperative levels because of the surgical trauma. There was no statistically significant difference in the three groups $(P>0.5)$. The WBC count gradually decreased in the experimental group, and after 3 weeks, it was close to normal. In contrast, the WBC count decreased only slowly in the control group and blank group, but it was still higher than normal at postoperative week 4 . The difference between the experimental group and other two groups was significant $(P<0.05)$. On the day before the debridement surgery, the level of $\mathrm{C}$-reactive protein was clearly high in the rabbit venous blood test because of the existence of osteomyelitis. On the third day after the debridement surgery, the amount of C-reactive protein in the three groups was not significantly different $(P>0.05)$ compared with the preoperative levels, due to the surgical trauma. The C-reactive protein gradually decreased in the experimental group, and at postoperative week 3 , it was close to normal levels. In the other two groups, it slowly decreased and was still higher than normal levels at postoperative week 4 . The difference between the experimental group and the other two groups was significant $(P<0.01$, Figure 5$)$. 

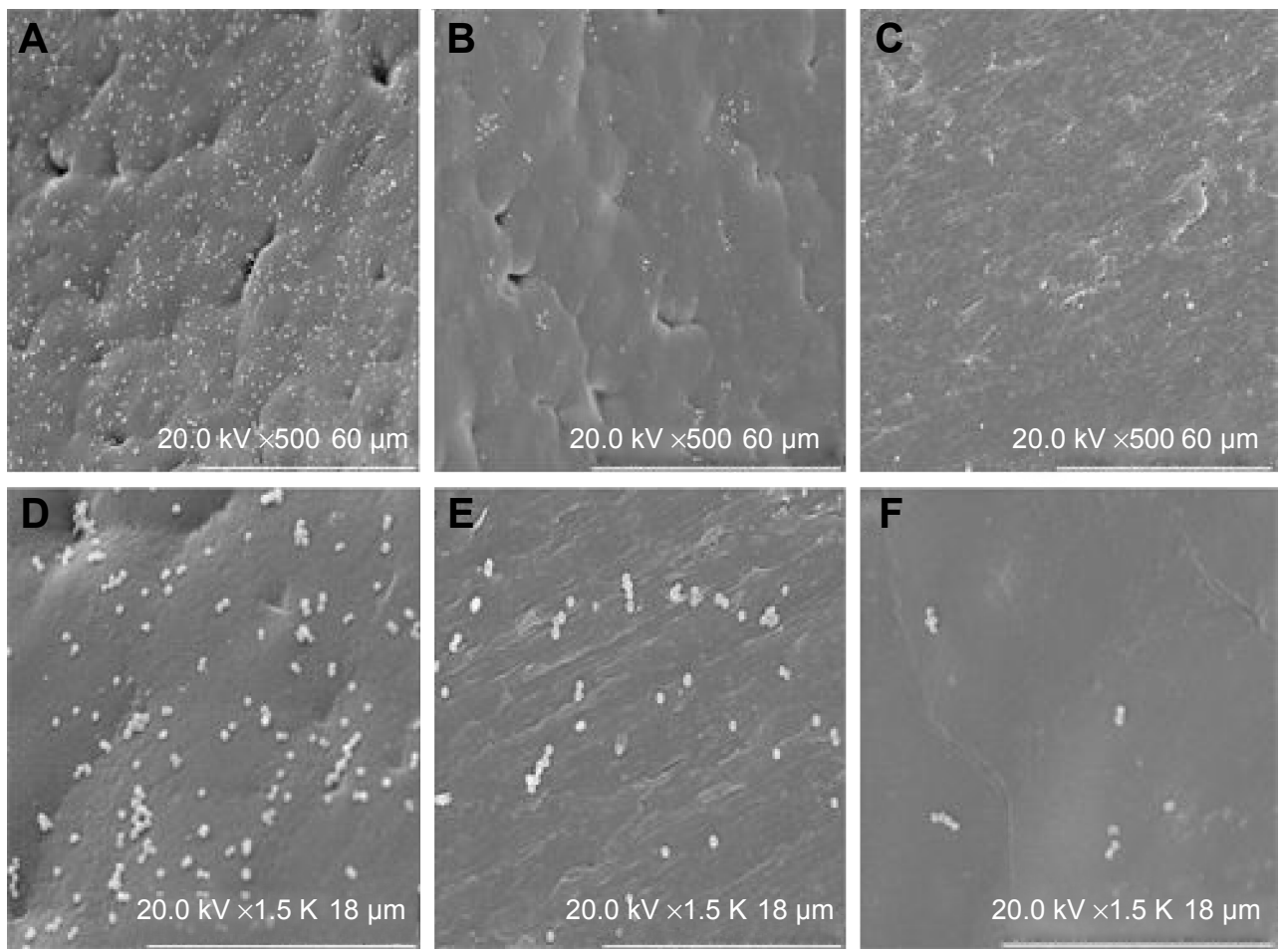

Figure 2 ATCC25923 was cultivated with three types of materials for 5 hours.

Notes: SEM: the first row $\times 500$, the second row $\times$ I,500. (A, D) no loaded medicine; (B, E) loaded with $2 \%$ RPT; (C, F) loaded with $4 \%$ RPT. The bacterial adhesion and growth were clearly different on the three kinds of materials.

Abbreviations: SEM, scanning electron microscopy; RPT, rifapentine.
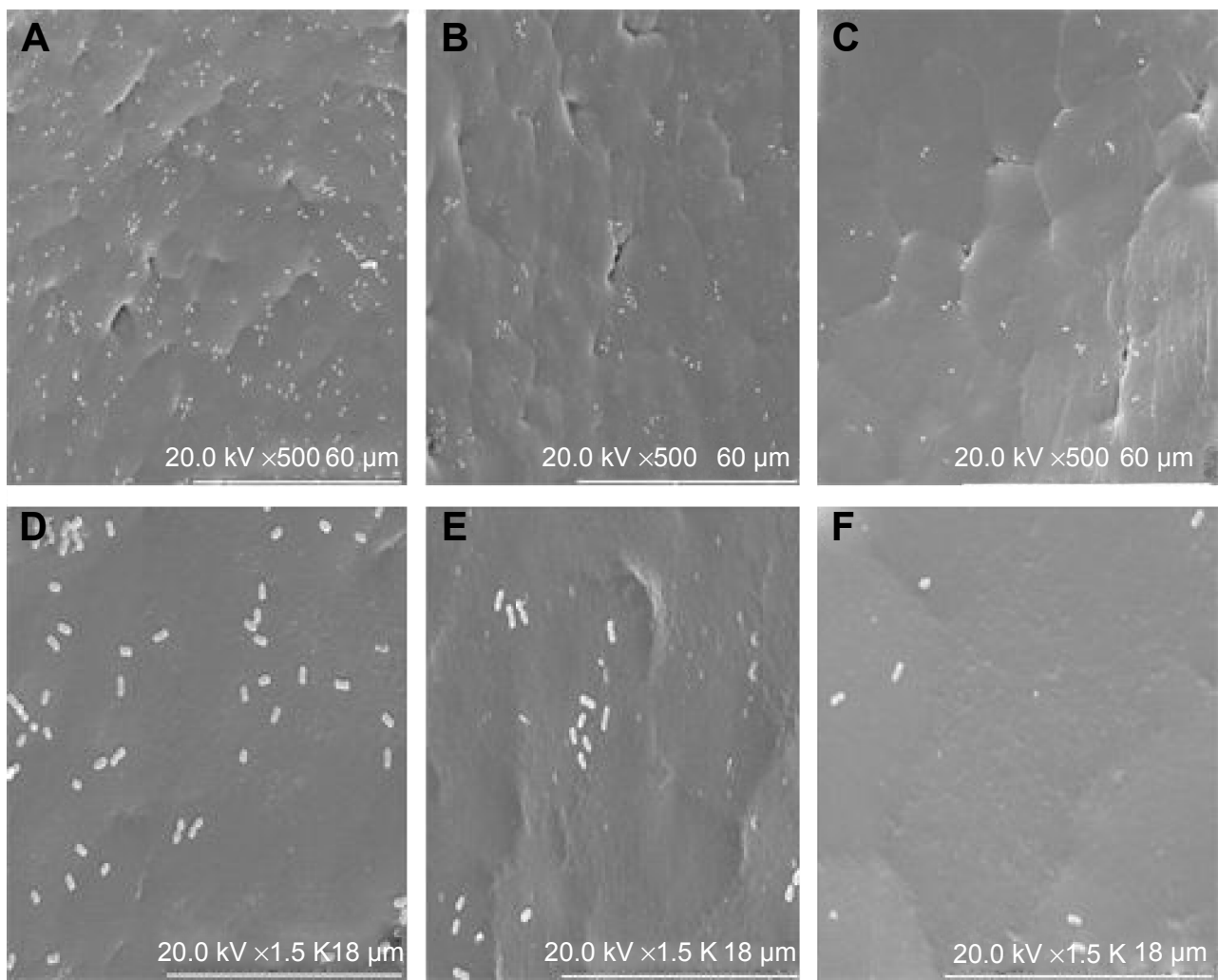

Figure 3 ATCC25922 was cultivated with three kinds of materials for 5 hours.

Notes: SEM: the first row $\times 500$, the second row $\times$ I,500). (A, D) no loaded medicine; (B, E) loaded with $2 \%$ RP; C, F - loaded with $4 \%$ RP. The bacterial adhesion and growth were clearly different on the three types of materials.

Abbreviations: SEM, scanning electron microscopy; RPT, rifapentine. 

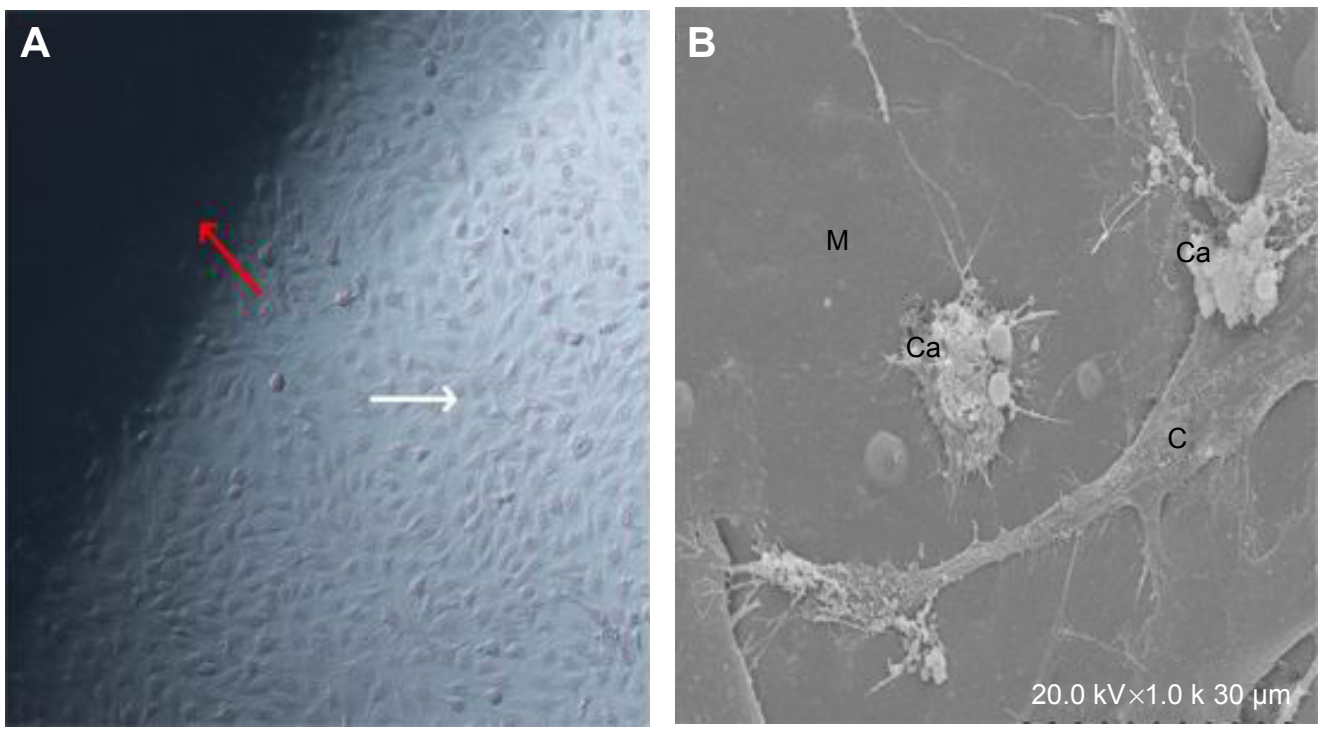

Figure 4 MG63 cells were cultivated with BHA for I week.

Notes: $(A)$ Inverted microscopy observation $(\times 100)$ shows a large amount of cell growth around the material. Red arrow is the material and white arrow is the MG63 cell. (B) SEM $(\times 1,000)$ shows many calcium nodules on the material. M, materials; Ca, calcium nodules; C, MG63 cell.

Abbreviations: BHA, bone-like hydroxyapatite; SEM, scanning electron microscopy.

A

Weight changes after the second surgery $(\mathrm{kg}, \overline{\mathrm{x}} \pm \mathrm{s}, \mathrm{n}=5)$

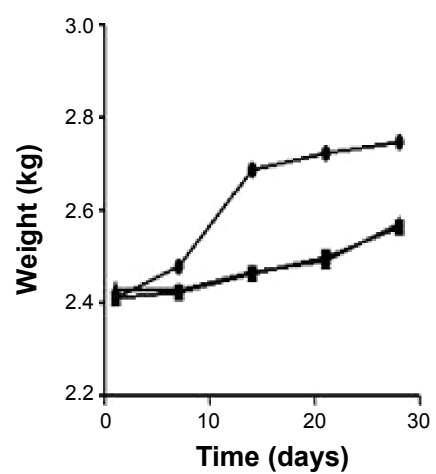

B

WBC changes after the second surgery $\left(10^{9}\right.$ cells $\left./ L, \bar{x} \pm s, n=5\right)$

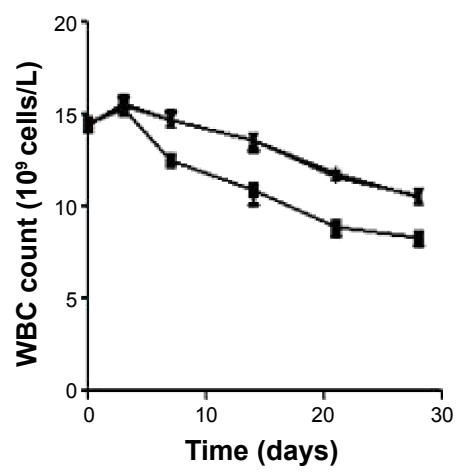

C

C-reactive protein changes after the second surgery ( $\mathrm{mg} / \mathrm{L}, \overline{\mathrm{x}} \pm \mathrm{S}, \mathrm{n}=5$ )

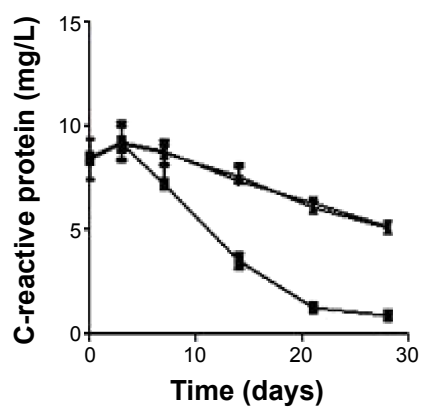

Experimental group

+ Control group

士 Blank group

Figure 5 Changes in weight $(\mathbf{A})$, WBC count $(\mathbf{B})$, and C-reactive protein $(\mathbf{C})$ after the debridement surgery and treatment.

Notes: The changes of weight, WBC count, and $C$-reactive protein were significantly different in the experimental group compared with the other two groups (weight, $P<0.05$;

WBC count and $C$-reactive protein, $P<0.01)$. Weight, WBC count, and $C$-reactive protein were not different in the control group compared with the blank group $(P>0.05)$. Abbreviation: WBC, white blood cells. 


\section{Radiological evaluation}

There was no animal death during modeling or during the treatment. Four weeks after the osteomyelitis induction, the right proximal tibia of all rabbits had clear chronic osteomyelitis on radiographic inspection with the following findings: reduced bone density, the trabecular bone being thin and sparse, dead bone, subperiosteal abscesses, and new periosteal bone formation. We confirmed that a chronic osteomyelitis animal model was successfully established, as described by Norden et al. ${ }^{18}$ The osteomyelitis model severity classification was level 3, 4 (including 9 in level 3 and 15 in level 4) using gross observation and radiological detection standards. ${ }^{20,21}$ After the debridement surgery, the radiographic results of animals in the three groups at week 12 showed that the proximal tibia shape had largely returned to the normal structure, that new trabecular bone had formed, and that the materials were mainly degraded and absorbed in the experimental group; whereas the shape of the proximal tibia was not recovered in the control or blank group, and showed the following deterioration trend: bone destruction, subperiosteal abscesses, and periosteal new bone formation. The carrier material was clearly visible in the control group (Figure 6).

\section{Pathological and immunohistochemical examination}

Four weeks after induction of osteomyelitis, the HE staining of bone tissue lesion showed a large number of clearly visible inflammatory cells, interstitial hemorrhage, fibrous hyperplasia, and bone necrosis extending from periosteum to bone marrow cavity. Grading the severity of osteomyelitis

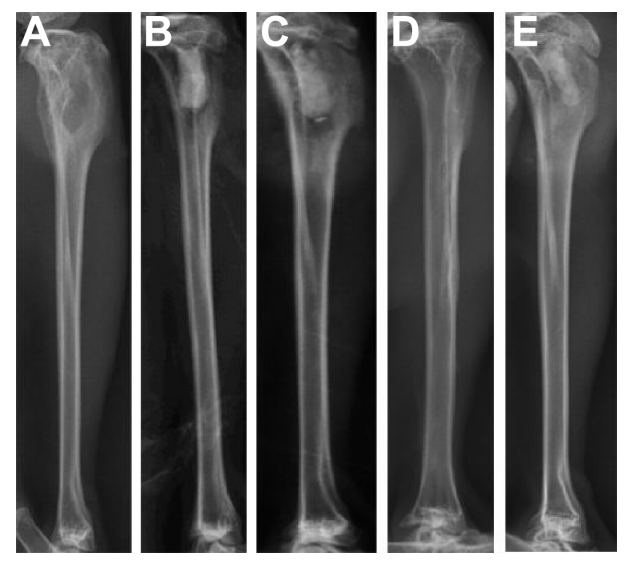

Figure 6 X-ray photograph inspections before and after treatment. Notes: (A) Four weeks after the induction of osteomyelitis, the proximal tibia has significant broadening, bone destruction, trabecular bone thinning, and periosteal hyperplasia. (B) and (C) show implants with the drug-loaded material and with no drug-loaded material, respectively, at day I. (D) After treatment with drug-loaded material for 12 weeks, the proximal tibia bone shape largely returned to normal, with faintly visible material. (E) After treatment with no drug-loaded material for 12 weeks, the proximal tibia damage was deteriorating, and the material was clearly visible, without new bone formation. using the methods described by Smeltzer et $\mathrm{a}^{19}$ includes four categories: acute inflammation, chronic inflammation, periosteal inflammation, and bone necrosis. At 12 weeks after the debridement surgery and treatment in the experimental group, inflammatory cells were not observed, most of the material was degraded and absorbed, and new bone and cartilage had formed. In contrast, inflammatory cells were clearly visible in the control and blank groups. In addition, the material was clearly visible due to inflammation and no new bone had formed in the control group (Figure 7). Twelve weeks after the debridement surgery and treatment, specimens were collected from the bone tissue lesion and stained using immunohistochemical techniques of collagen fiber. The formation of collagen fibers was clearly visible in the the experimental group around the carrier materials, whereas the formation was not observed in the control group (Figure 8).

\section{Microbial examination}

Four weeks after induction of osteomyelitis, the bacterial culture results of lesion tissue showed an extensive bacterial growth on the culture plate, and G-staining and coagulase test confirmed the bacteria as $S$. aureus. Four weeks after the debridement surgery and treatment, bone tissue lesions were collected from the three groups and cultivated for bacteria, and then the bacterial $\mathrm{CFU} / \mathrm{g}$ bone was calculated. A lower CFU/g was observed in the experimental group $(2.8 \times 10 \pm 1.4 \times 10 \mathrm{CFU} / \mathrm{g})$, and the concentration of bacteria was more in the control and blank groups $\left(5.0 \times 10^{5} \pm 1.2 \times 10^{5} \mathrm{CFU} / \mathrm{g}\right.$ and $5.3 \times 10^{5} \pm 1.2 \times 10^{5} \mathrm{CFU} / \mathrm{g}$, respectively). The difference between the experimental group and the other two groups was significant $(P<0.01$, Table 1).

\section{Discussion}

The clinical treatment of chronic osteomyelitis induced by $S$. aureus is extremely difficult, particularly involving MRSA. S. aureus in the infective site causes ischemia and necrosis of the bone and the surrounding tissue, and it causes the formation of a biofilm that blocks blood flow and prevents antibiotic penetration. ${ }^{22} S$. aureus simultaneously produces coagulase on its surface, which results in a state of immune tolerance that allows the bacteria to escape removal by inflammatory cells and immune cells. In addition, S. aureus is resistant to multiple antibiotics, which can lead to persistent infection. In summary, it is difficult to achieve effective antibiotic drug concentrations at the local site by intravenous injection or oral routes, which makes the treatment effect not ideal. ${ }^{23}$ 

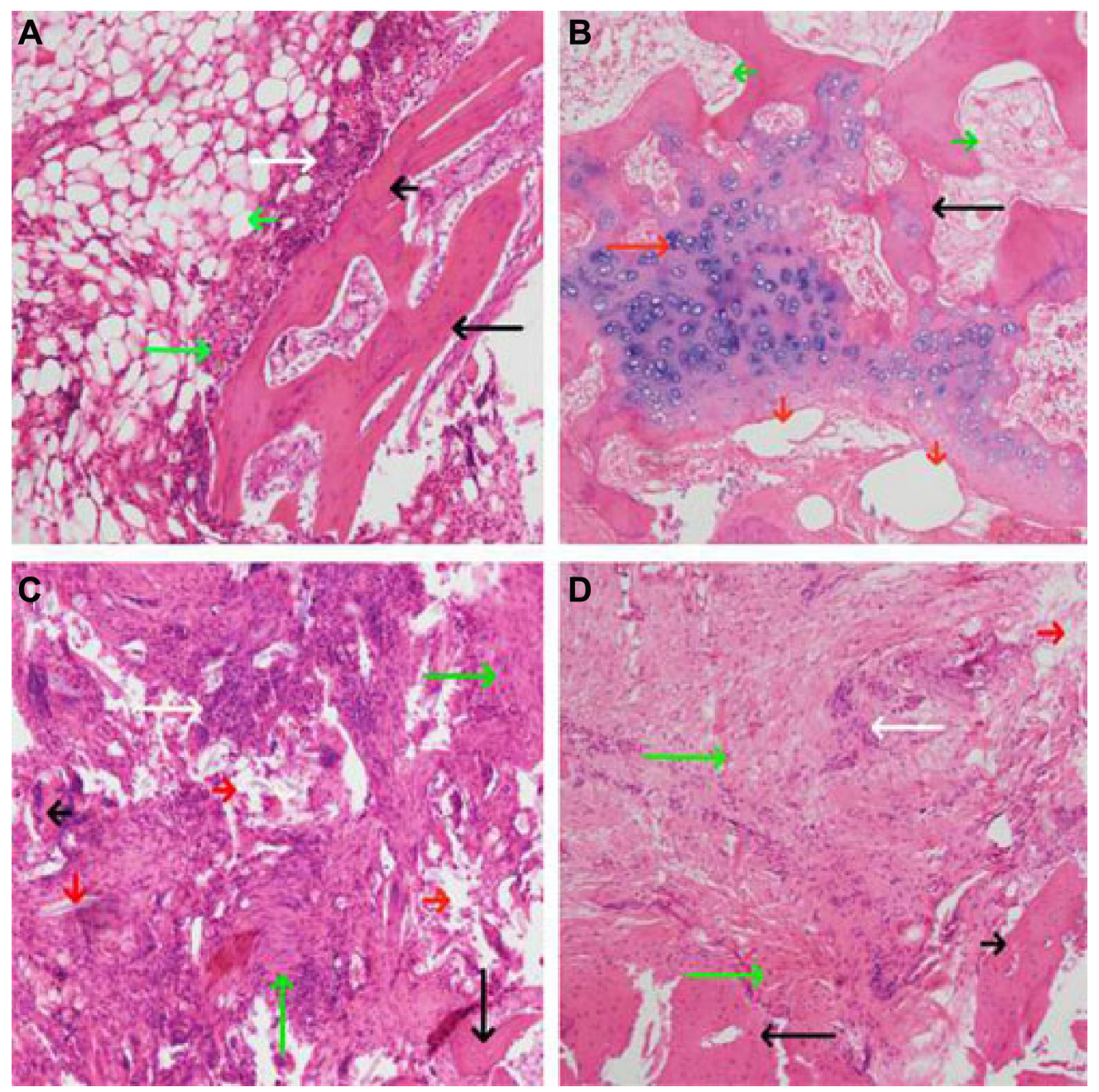

Figure 7 HE staining of bone tissue lesion $(\times 100)$.

Notes: (A) Four weeks after the induction of chronic osteomyelitis, there was clearly visible inflammatory cell infiltration, fibrous hyperplasia, dead bone from periosteum to bone marrow cavity. (B-D) Treatment of osteomyelitis for 12 weeks. (B) In the drug-loaded group, the new bone and cartilage formed and materials were largely absorbed. (C) and (D), no drug-loaded group and blank group, respectively, inflammatory cells, fibrous hyperplasia, and dead bone were clearly visible, and new bone did not form. Long white arrow, inflammatory cells; short white arrow, bleeding; long black arrow, trabecular bone; short black arrow, dead bone; long red arrow, cartilage and chondrocytes; short red arrow, materials long green arrow, fibrous hyperplasia and necrosis; short green arrow, the tissue of medullary cavity.

Abbreviation: $\mathrm{HE}$, hematoxylin and eosin.
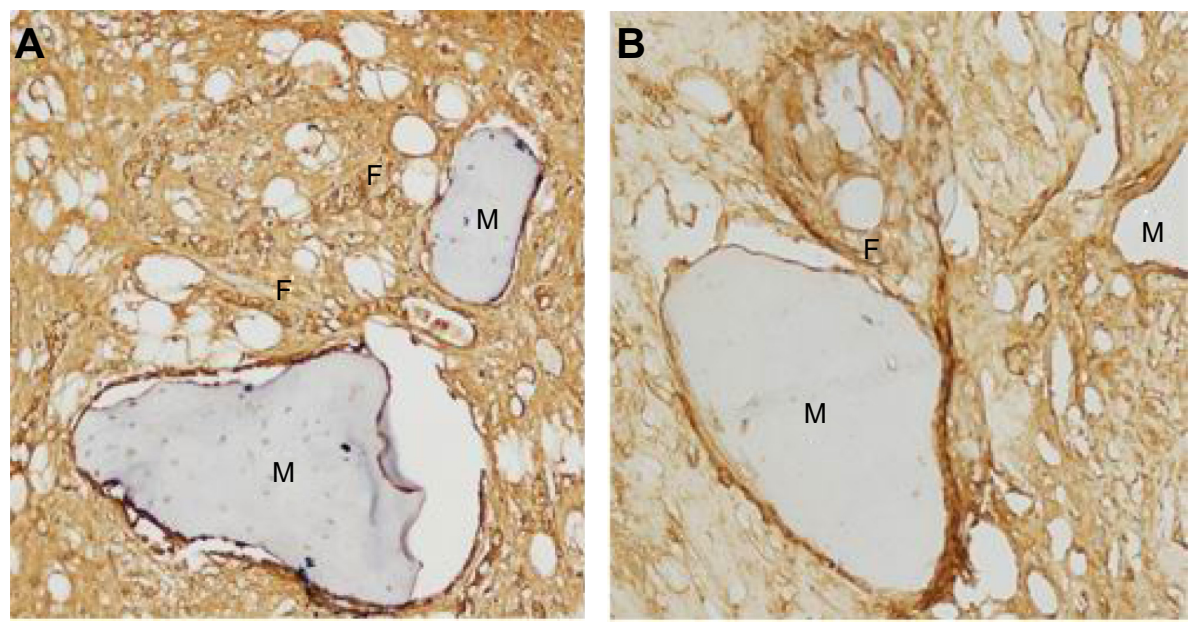

Figure 8 Collagen fiber immunohistochemical examination of the bone tissue lesion $(\times 200) 12$ weeks after the second surgical implantation of materials.

Notes: (A) Drug-loaded group, a large number of collagen fibers formed around the materials. (B) No drug-loaded groups, collagen fiber formation was not notable, only a small amount of formation was observed. F, collagen fiber; M, material. 
Table I Bacteria count per gram of bone tissue in each group (CFU/g, $\bar{x} \pm s, n=4)$

\begin{tabular}{llll}
\hline No & $\begin{array}{l}\text { Experimental } \\
\text { group }\end{array}$ & $\begin{array}{l}\text { Control } \\
\text { group }\end{array}$ & $\begin{array}{l}\text { Blank } \\
\text { group }\end{array}$ \\
\hline 1 & $3.0 \times 10$ & $6.4 \times 10^{5}$ & $5.8 \times 10^{5}$ \\
2 & $1.2 \times 10$ & $4.2 \times 10^{5}$ & $4.6 \times 10^{5}$ \\
3 & $2.4 \times 10$ & $5.6 \times 10^{5}$ & $6.8 \times 10^{5}$ \\
4 & $4.6 \times 10$ & $3.8 \times 10^{5}$ & $4.0 \times 10^{5}$ \\
Mean \pm SD & $2.8 \times 10 \pm 1.4 \times 10$ & $5.0 \times 10^{5} \pm 1.2 \times 10^{5}$ & $5.3 \times 10^{5} \pm 1.2 \times 10^{5}$ \\
\hline
\end{tabular}

Note: The experimental group was compared with control and blank groups $(P<0.01)$.

Abbreviations: CFU, colony forming units; SD, standard deviation.

In the 1970s, Buchholz and Engelbrecht ${ }^{3}$ and Wahlig et $\mathrm{al}^{4}$ successively reported that the application of antibiotics in combination with PMMA bone cement effectively treated osteomyelitis. However, this material is nondegradable, shows no biological activity, and has many disadvantages. ${ }^{5-7}$ Currently, we have materials that possess good biocompatibility, biodegradable absorption, biological activity, osteogenic activity, and controllable release of materials. These advantages have contributed to the great progress in the treatment of chronic osteomyelitis. Antibiotics are released slowly from these materials at the local infection site to effectively eradicate the bacterial infection.

RPT is a new type of rifamycin derivative, and its antibacterial spectrum is similar to that of rifampin. It has sterilization ability for tuberculosis bacilli, and it also has bacterial inhibitory and sterilization effects for Gram-positive cocci and Gram-negative bacilli, including $S$. aureus. ${ }^{15}$ It can enter into the biofilms formed by bacteria and into the inflammatory cells to kill the bacteria. RPT has stronger antibacterial activity and fewer side effects than rifampin, and has a longer drug elimination half-life of up to $14-15$ hours. ${ }^{24}$ In vitro, the minimal inhibitory concentration of RPT is lower than that of rifampicin. ${ }^{25}$ Animal studies have shown that RPT concentration was significantly higher in the bone tissue than in the plasma, ${ }^{26}$ and this capacity could allow for penetration into infected and dead bone to kill bacteria. In addition, its ability to penetrate is stronger than that of vancomycin, ${ }^{15}$ and it can penetrate into infected bone, dead bone, biofilm, and inflammatory cells to effectively eradicate bacterial infections, particularly in anaerobic environments. RPT enters into inflammatory cells to kill bacteria and eradicate bone and soft tissue infection, thereby preventing the recurrence of chronic osteomyelitis.

In this study, BHA/PAA was used as a carrier of RPM to prepare sustained-release drug materials for the treatment of rabbit tibial chronic osteomyelitis. The microporous nature of BHA/PAA and PLGA, their gradual degradation, and release of RPT in a slow, continuous, and stable manner at the infection site contributed to achieve effective drug concentrations, to eradicate the bacterial infection, and successfully cure chronic osteomyelitis.

The antibacterial activity of the drug-loaded materials was evaluated through in vitro and in vivo experiments. In vitro, the bacterial inhibition experiments (Figure 1) confirmed that the drug-loaded materials inhibited $S$. aureus growth for up to 5 weeks. This finding confirmed that the materials were able to slowly release antibiotics and effectively inhibit bacterial growth. In vitro, the antibacterial experiments (Figures 2 and 3 ) showed that bacterial adhesion and growth was clearly visible through SEM. These parameters were significantly reduced in the drug-loaded groups, and there was variability based on the different doses of drug loaded. Antibacterial activity was also examined in vivo 4 weeks after the debridement surgery and treatment. The bone tissues, collected from the experimental, control and blank groups, were cultivated for bacteria, and the bacterial colony count was calculated. The bacterial colony counts were extremely low in the experimental group. However, in the other two groups, the bacterial colony counts were extremely high (Table 1). After treatment for 12 weeks, HE pathology staining of the bone tissue lesion was performed and no inflammatory cells were observed in the experimental group. However, inflammatory cells were clearly visible in the other two groups (Figure 7). Furthermore, the change in the levels of C-reactive protein was measured. C-reactive protein is an important index of inflammation and stress. If C-reactive protein falls quickly, it indicates that inflammation is improving; however, if it falls slowly or is continuously high, it indicates that inflammation is persisting. This value tended to be normal after 2 weeks in the experimental group; however, it slowly decreased in the control and blank groups and was still higher than normal at 4 weeks. It indirectly showed that the drug-loaded materials eradicated the bacterial infection (Figure 5). The results of the in vivo antibacterial experiment confirmed that RPM-loaded BHA/PAA effectively eradicated the bacterial infection and cured chronic osteomyelitis. In vitro and in vivo, the studies mentioned earlier also showed that the RPM-loaded BHA/PAA slowly and continuously released antibiotics to effectively kill bacteria.

BHA is a kind of carbonate HA which has a composition and structure approximating similar to that of natural bone. In BHA, there is lattice distortion of HA because of the presence of $\mathrm{CO}_{3}^{2-}$ instead of $\mathrm{PO}_{4}^{3-}$ or $\mathrm{OH}^{-}$in the structure, resulting in a large number of internal defects in the HA crystal. Its crystal formation is incomplete with a low degree of crystallinity; hence, BHA is easily degraded and absorbed in the body, which helps in retaining the original 
good biocompatibility, biological activity, and osteogenic effect of $\mathrm{HA}^{27} \mathrm{BHA}$ exchanges $\mathrm{Ca}^{2+}, \mathrm{OH}^{-}, \mathrm{HPO}_{4}{ }^{2-}, \mathrm{PO}_{4}{ }^{3-}$, and $\mathrm{CO}_{3}^{2-}$ through autolysis in the body fluid when it is implanted in the body, and it is bound with the host bone as a whole through hydrogen bonding. It provides an excellent physiological scaffold for new bone formation and regeneration. In addition, BHA gradually degrades during the process of bone healing until the defected bone is entirely replaced. Because the material is naturally porous and has a large surface area, it is conducive to new blood vessel growth and formation, ${ }^{28}$ which increases the ability of local tissue blood supply to tissues and improves defence against inflammation. BHA also is also conducive to osteoblast and bone marrow stem cell adhesion, proliferation, growth, as well as to the formation of new bone tissue on the material. ${ }^{29,30}$ Additionally, nanocrystals of BHA are smaller than those of HA, so their surface area is increased, which increases the surface area and hence the drug-loading capacity. This allows for the achievement of high local antibiotics concentrations of antibiotics in a short period of time when implanted in the body, which results in the effective killing of pathogens in the infective lesions. However, pure BHA is highly brittle, and hence is not conducive to processing and plasticity. When it combines with PAA, its toughness and mechanical property is greatly enhanced and provides good support for bone defects. PAA, with a similar molecular structure to collagen, has good biocompatibility and is biodegradable, ${ }^{9}$ and its degradation products includes glycine, proline, and hydroxyproline, which promote the formation of collagen fibers. Collagen fibers are the scaffold of bone formation and attract calcium deposits, which promotes new bone formation. Preliminary experiments $^{31}$ had shown that the BHA/PAA material had good biological safety, compatibility, and bone conductibility.

For the in vitro osteogenesis experiments, MG63 cells were cultivated together with BHA/PAA for 7 days. Inverted microscopy observation showed that a large number of cells adhered and grew on the material. Under SEM, extensive cell adhesion, growth, and calcium nodule formation was visible on the material. These results showed that the material had good biocompatibility, biological activity, and osteogenic activity (Figure 4 ).

The degradation and osteogenic ability of the RPMloaded BHA/PAA material was evaluated in vivo. After 12 weeks of treatment, I collagen fibers were detected in the bone tissue lesions via through immunohistochemical staining. A large number of I collagen fibers formed around the materials in the experimental group. However, there was no or only a small amount of I collagen fiber formation in the control group (Figure 8), due to persistent inflammation.
Collagen fibers are a scaffold for calcium salt deposition and the formation of new bone. Studies have shown that collagen fibers are important indicators of reflexion bone formation, and they play an important role in calcium nodule formation and osteoblast adhesion, differentiation, and maturation. ${ }^{32}$ HE staining showed that most of the material was degraded and new trabecular bone and cartilage formed in the experimental group of containing drug-loaded material. In contrast, degradation of material and the formation of new bone were not observed in the group where no drug was loaded into the material (Figure 7), due to persistent inflammation. The results of immunohistochemical staining for collagen fibers and HE staining confirmed that the RPM-loaded BHA/PAA had good biological activity and osteogenesis induction ability, promoting new bone formation and rebuilding the bone defect with gradual degradation and absorption.

For radiographic detection, 24 rabbits with chronic osteomyelitis were conducted by debridement surgery. The results of the treatment confirmed that the bone shape gradually improved in the RPM-loaded BHA/PAA experimental group, and it largely returned to normal at 12 weeks. The material was faintly visible, thus showing that the material was largely degraded and absorbed. However, in the BHA/ PAA control group, the bone shape was not improved, and there was a deterioration trend with clearly visible material, which indicated no degradation and absorption (Figure 6). The imaging examination results showed that the drug-loaded material eradicated the bacterial infection, promoted new bone formation, and rebuilt the bone defect.

\section{Conclusion}

Through in vitro and in vivo antibacterial experiments, this study confirmed that RPM-loaded BHA/PAA effectively restrained and killed bacteria, and controlled rabbit tibial infection. The material slowly and continuously released the antibiotic, RPT, which had an effective sterilization effect against $S$. aureus. In vitro, the osteogenesis experiments showed that BHA/PAA promoted MG63 adhesion, aggregation, differentiation, and calcium nodule formation. In vivo, the osteogenesis experiments showed that RPMloaded BHA/PAA induced osteogenesis, promoted new bone formation, and reconstructed bone defects while the material was gradually degraded and absorbed. In conclusion, the current study shows that RPM-loaded BHA/PAA is effective for the treatment of chronic osteomyelitis and for the reconstruction of bone defects. This method could be a promising method for the treatment of orthopedic infections induced by MRSA and should be investigated further in future research and clinical work. 


\section{Acknowledgment}

This study was financially supported by the National Natural Science Foundation Program of China (81171685).

\section{Disclosure}

The authors report no conflicts of interest in this work.

\section{References}

1. Lew DP, Waldvogel FA. Osteomyelitis. Lancet. 2004;364:369-379.

2. Sia IG, Berbari EF. Osteomyelitis. Best Pract Res Clin Rheumatol. 2006;20:1065-1081.

3. Buchholz HW, Engelbrecht H. Depot effects of various antibiotics mixed with Palacos resins. Chirurg. 1970;41:511-515.

4. Wahlig H, Dingeldein E, Bergmann R, Reuss K. The release of gentamicin from polymethylm ethacrylate beads. An experimental and pharmacokinetic study. J Bone Joint Surg Br. 1978;60-B:270-275.

5. Wong MW, Hui M. Development of gentamicin resistance after gentamicin-PMMA beads for treatment of foot osteomyelitis: report of two cases. Foot Ankle Int. 2005;26:1093-1105.

6. Gürsel I, Korkusuz F, Türesin F, Alaeddinoglu NG, Hasirci V. In vivo application of biodegradable controlled antibiotic release systems for the treatment of implant-related osteomyelitis. Biomaterials. 2001;22:73-80.

7. Wei G, Kotoura Y, Oka M, et al. A bioabsorbable delivery system for antibiotic treatment of osteomyelitis: the use of lactic acid oligomer as a carrier. J Bone Joint Surg Br. 1991;73:246-252.

8. Genile P, Chiono V, Boccafoschi F. Composite films of gelatin and hydroxyapatite/bioactive glass for tissue-engineering applications. J Biomater Sci Polym Ed. 2010;21(8/9):1207-1226.

9. Roohani-Esfahani SI, Nour-iKhorasani S, Lu Z, Appleyard R, Zreiqat $\mathrm{H}$. The influence hydroxyapatite nanoparticle shape and size on the properties of biphasic calcium phosphate scaffolds coated with hydroxyapatite-PCL composites. Biomaterials. 2010;31:5498-5509.

10. StallmannHP, FaberC, Bronckers AL, Nieuw Amerongen AV, Wuisman PI. In vitro gentamicin release from commercially available calciumphosphate bone substitutes influence of carrier type on duration of the release profile. BMC Musculoskelet Disord. 2006;7:18.

11. Koort JK, Suokas E, Veiranto M, et al. In vitro and in vivo testing of bioabsorbable antibiotic containing bone filler for osteomyelitis treatment. J Biomed Mater Res A. 2006;78:532-540.

12. Orhan Z, Cevher E, Mülazimoglu L, et al. The preparation of ciprofloxacin hydrochloride-loaded chitosan and pectin microspheres: their evaluation in an animal osteomyelitis model. J Bone Joint Surg Br. 2006;88:270-275.

13. Wu Y, Bose S. Nanocrystalline hydroxyapatite: micelle templated synthesis and characterization. Langmuir. 2005;21:3232-3234.

14. Freitas S, Merkle HP, Gander B. Microencapsulation by solvent extraction/ evaporation: reviewing the state of the art of microspheres preparation process technology. J Control Release. 2005;102:313-332.

15. Petri WA. Chemotherapy of tuberculosis, Mycobacterium avium complex disease, and leprosy. In: Goodman LS, Gilman A, Brunton LL, Lazo JS, Parker KL, editors. Goodman and Gillman's The Pharmacological Basis Of Therapeutics, 11 th ed. New York: Churchill Livingstone. 2006;1207-1210.

Drug Design, Development and Therapy

\section{Publish your work in this journal}

Drug Design, Development and Therapy is an international, peerreviewed open-access journal that spans the spectrum of drug design and development through to clinical applications. Clinical outcomes, patient safety, and programs for the development and effective, safe, and sustained use of medicines are a feature of the journal, which

Submit your manuscript here: http://www.dovepress.com/drug-design-development-and-therapy-journa
16. Peng X, Li Y, Wang X, et al. Medical nano hydroxyapatite/polyamide 66 composite material in vitro immersion behavior research. J Funct Mater. 2004;2:253-255.

17. O'Reilly T, Mader JT. Rat model of bacterial osteomyelitis of the tibia. In: Zak O, Sande MA, editors. Handbook of Animal Models of Infection: Experimental Model in Antimicrobial Chemotherapy. San Diego, CA: Academic Press. 1999;560-575.

18. Norden CW, Myerowitz RL, Keleti E. Experimental osteomyelitis due to Staphylococcus aureus or Pseudomonas aeruginosa. A radiographicpathological correlative analysis. Br J Exp Path. 1980;61:451-460.

19. Smeltzer MS, Thomas JR, Hickmon SG, et al. Characterization of a rabbit model of Staphylococcal osteomyelitis. J Orthop Res. 1997;15:414-421.

20. Shirtliff ME, Calhoun JH, Mader JT. Experimental osteomyelitis treatment with antbiotic impregnated hydroxyapatite. Clin Orthop Relat Res. 2002;40:239-247.

21. Mader JT, Stevens CM, Stevens JH, Ruble R, Lathrop JT, Calhoun JH. Treatment of experimental osteomyelitis with a fibrin sealant antibiotic implant. CIin Orthop Relat Res. 2002;403:58-72.

22. Costerton JW. Biofilm theory can guide the treatment of device-related orthopaedic infections. Clin Orthop Relat Res. 2005;437:7-11.

23. Stewart PS, Costerton JW. Antibiotic resistance of bacteria in biofilms. Lancet. 2001;358:135-138.

24. Keung AC, Eller MG, McKenzie KA, Weir SJ. Single and multiple dose pharmacokinetics of rifapentine in man: part II. Int J Tuberc Lung Dis. 1999;3:437-444.

25. Venkataraman P, Paramasivan CN, Prabhakar R. In vitro activity of rifampicin, rifapentine and rifabutin against south Indian isolates of Mycobacterium tuberculosis. Indian J Tuberc. 1993;40:17-20.

26. Assandri A, Ratti B, Cristina T. Pharmacokinetics of rifapentine, a new long lasting rifamycin, in the rat, the mouse and the rabbit. $J$ Antibiot (Tokyo). 1984;37:1066-1075.

27. Gentile P, Chiono V, Boccafoschi F, et al. Composite films of gelatin and hydroxyapatite/bioactive glass for tissue-engineering applications. J Biomater Sci Polym Ed. 2010;21:1207-1226.

28. Chanda A, Singha Oy R, Xue W, Bose S, Bandyopadhyay A. Bone cell-materials interaction on alumina ceramics with different grain sizes. Mater Sci Eng C. 2009;29:1201-1206.

29. Mastrogiacomo M, Scaglione S, Martinetti R, et al. Role of scaffold internal structure on in vivo bone formation in macroporous calcium phosphate bioceramics. Biomaterials. 2006;27:3230-3237.

30. Turco G, Marsich E, Bellomo F, et al. Alginate/hydroxyapatite biocomposite for bone ingrowth: a trabecular structure with high and isotropic connectivity. Biomacromolecules. 2009;10:1575-1583.

31. Dai Y, Li J, Zhao ZH, et al. Nano amino apatite/poly (amino acid composite safety evaluation. J Third Military Medical University. 2010;32: 294-299.

32. Yang ZM, Yu XJ, Huang FG, et al. Exogenous type I collagen of embryonic periosteum ossification cell biological characteristics influence. Journal of West China Major Medical Journal. 2001;32:1. has also been accepted for indexing on PubMed Central. The manuscript management system is completely online and includes a very quick and fair peer-review system, which is all easy to use. Visit http://www.dovepress.com/testimonials.php to read real quotes from published authors. 